\title{
Can High Dose Metamizol Medication Contribute to Postsurgical Hemorrhage: A Case Report
}

\author{
Dagmar Wildpaner'1, Beat Flueckiger ${ }^{1}$, Urs Dieter Schmid², Dominik Weishaupt ${ }^{3}$, \\ Robert Theiler ${ }^{1}$ \\ ${ }^{1}$ Department of Rheumatology, Stadtspital Triemli, Zurich, Switzerland \\ ${ }^{2}$ Division of Neurosurgery, Stadspital Triemli, Zurich, Switzerland \\ ${ }^{3}$ Department of Radiology, Stadtspital Triemli, Zurich, Switzerland \\ Email: dagmar.wildpaner@triemli.zuerich.ch
}

Received 14 February 2015; accepted 6 April 2015; published 14 April 2015

Copyright (C) 2015 by authors and Scientific Research Publishing Inc.

This work is licensed under the Creative Commons Attribution International License (CC BY). http://creativecommons.org/licenses/by/4.0/

(c) (i) Open Access

\begin{abstract}
The use of metamizol significantly increased in the last decade, especially in non US countries [1]. Although metamizol is known to be a non selective COX inhibitor and therefore has platelet inhibiting potential, it is widely used in pain management in postsurgical setting. Methods: We present a case of bleeding after microsurgical decompression of a narrow lumbar spinal canal. Our patient developed a bleeding complication 3 days after the surgery. The bleeding spontaneously resolved after stopping the application of metamizol. Metamizol is a non selective COX inhibitor and is used as standard analgetic drug after surgery in our department, whereas other COX inhibitors are not. We made an intensive literature research to evaluate the prohaemorrhagic potential of metamizol. Metamizol has proven dose dependent platelet inhibiting effect. Conclusions: This is a quite ordinary case and there is no proof of metamizol being responsible for the bleeding in this case, it still poses questions at our daily routine and the question about a dose dependent clinically relevant prohaemorrhagic effect of metamizol remains unanswered. A proper study is needed.
\end{abstract}

\section{Keywords}

Metamizol, Narrow Lumbar Spinal Canal, Postsurgical Analgesia, Bleeding Complication

\section{Introduction}

The more than 90-year-old drug metamizol, also known as dipyrone, was taken from the market in many coun-

How to cite this paper: Wildpaner, D., Flueckiger, B., Schmid, U.D., Weishaupt, D. and Theiler, R. (2015) Can High Dose Metamizol Medication Contribute to Postsurgical Hemorrhage: A Case Report. Open Journal of Modern Neurosurgery, 5, 53-58. http://dx.doi.org/10.4236/ojmn.2015.52009 
tries due to cases of severe agranulocytosis. In some other countries, especially in Europe and Latin America, it is still widely used as an analgesic and antipyretic drug and in some places it is even available over the counter without prescription [2]. The use of this drug has multiplied in the last years as it is assumed to be safe.

Metamizol is an antipyretic analgesic introduced into clinical practice in 1922. The water-soluble pyrazolinone derivative is available in oral, rectal, and injectable forms. It is rapidly hydrolyzed in the gastric juice to its main and active metabolite 4-methyl-amino-antipyrine [3].

The mode of action is still not entirely clear. Apart from a minor central effect metamizol and its metabolites act mainly via both COX 1 and COX 2 inhibition [3] [4]. In vitro studies showed that the prohaemorrhagic effect of metamizol was comparable with the one of aspirin [5]. In contrast to classical COX inhibitors metamizol is a stronger inhibitor of platelet aggregation [5], but has no anti-inflammatory effect and low gastrointestinal toxicity, indicating a different mode of action [2].

\section{Case Report}

\section{Patients Consent Obtained}

We present a case of a 72-year-old female patient with severe multilevel lateral recess stenosis at L2/3 - L4/5, who developed muscular weakness in the quadriceps muscle and therefore underwent microsurgical bilateral decompression of three segments via three unilateral laminotomies.

Additionally the patient had various comorbidities in her medical records. The acetylsalicylic acid medication, prescribed from her cardiologist because of a coronary heart disease, was discontinued following our routine protocol 7 days before the surgery was scheduled. The operation itself was uneventful. The patient received general anesthesia with systemic opioid analgesics. An interlaminar access was performed at level L2/3, L3/4 and $\mathrm{L} 4 / 5$, the rezessal nerves were released by removing parts of the ligamentum flavum and the internal surface of the hemi-laminae. Perfect local haemostasis was performed as usual, and no Redon drain was inserted therefore. Due to early mobilization and apart from age no additional risk factors, she got no thrombosis prophylaxis according the 2009 guidelines of the Association of the Scientific Societies in Germany [6]. In the first day the postoperative course was uneventful. The analgesic regime was prescribed according to our local routine, containing opioids, $4 \mathrm{~g}$ paracetamol and up to $4 \mathrm{~g}$ metamizol in 4 single doses per day and the patient was not anticoagulated to prevent thromboembolic issues. Our patient recovered quickly and the analgesics could be reduced, leaving only metamizol and low dose opioids. The patient was already fully mobilized. On day 3 the local pain increased again and at the same time it became increasingly radicular. Due to calf pain she received $40 \mathrm{mg}$ enoxaparine as a single dose that was stopped thereafter as soon as a deep venous thrombosis was ruled out via compression ultrasound. On the same day we performed an MRI of the spine and discovered a big epidural hemorrhage on L2/3 to L4/5 with compression of the dural sac (Figure 1).
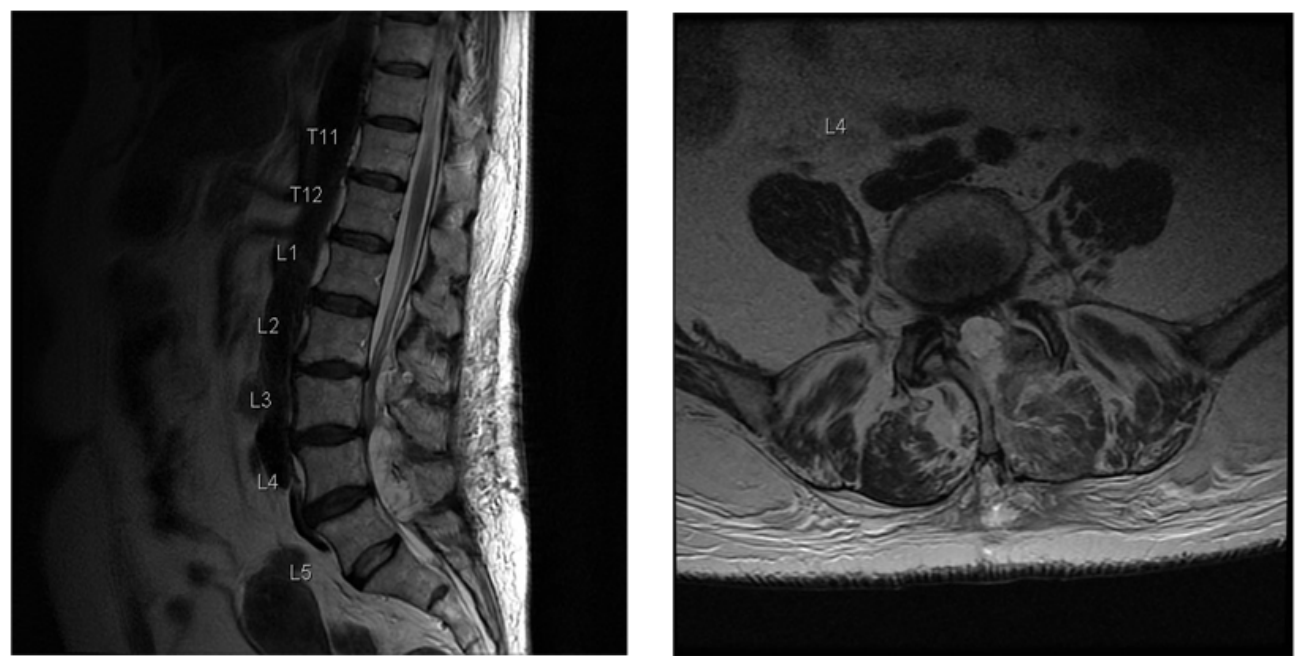

Figure 1. T2-weighted MR images of the lumbar spine 4 days after surgery. Sagittal and axial MR images at L4/5 level demonstrate an acute multisegmental epidural bleeding between L2/3 to L4/5 levels with almost complete obliteration of the spinal canal and severe compression of the dural sac. 
We browsed the medical records to find drugs with prohaemhorragic potential and found aspirin $100 \mathrm{mg}$ per day, which was stopped 7 days prior the operation and metamizol, which she got in high doses as analgesic treatment. The enoxaparin she got only once after symptom onset, so we considered it not causal for the bleeding. All medications with prohaemorrhagic potential were stopped and the patient was treated conservatively with high doses of steroids. The pain could be managed that way and surgical decompression was not necessary.

In the next weeks our patient recovered slowly. MRIs were performed and showed a slow reabsorption of the haematoma (Figure 2 and Figure 3). Clinically at the 3-month control the patient had no neurological signs or symptoms (such as radicular pain, neuropathic pain or paresthesia) and no signs of polyradiculo(neuro)pathy.

\section{Discussion}

This is not an extraordinary case. It still poses different questions at our routine protocols especially in non US countries. In this particular case there are at least four other potential reasons for the bleeding: mechanical surgery related causes, acetylsalicylic acid, enoxaparin and metamizol medication.

The patient received enoxaparin (once $40 \mathrm{mg}$ on day 3) after reporting pain in her lower limb. In retrospective, this pain was caused by the haematoma. While not being causal for the development, it might have contributed to the extent of the bleeding.
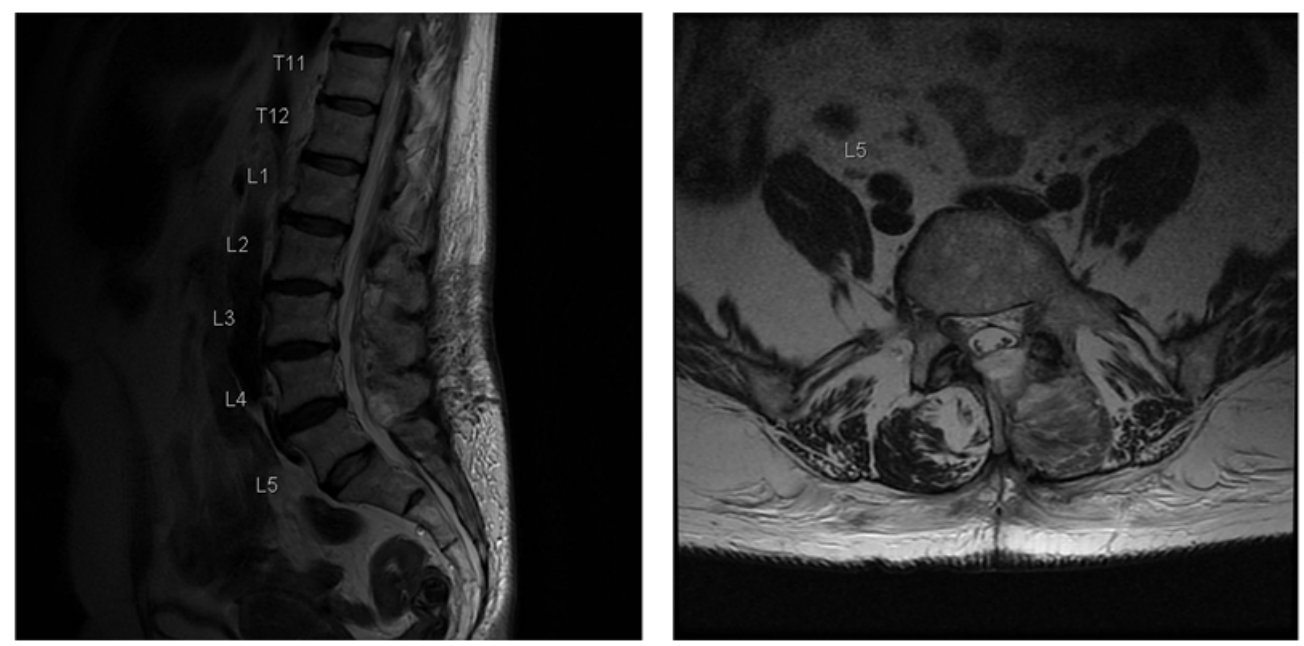

Figure 2. T2-weighted MR images of the lumbar spine in sagittal and axial planes 3 weeks after surgery. There is considerable regression of the epidural hematoma.
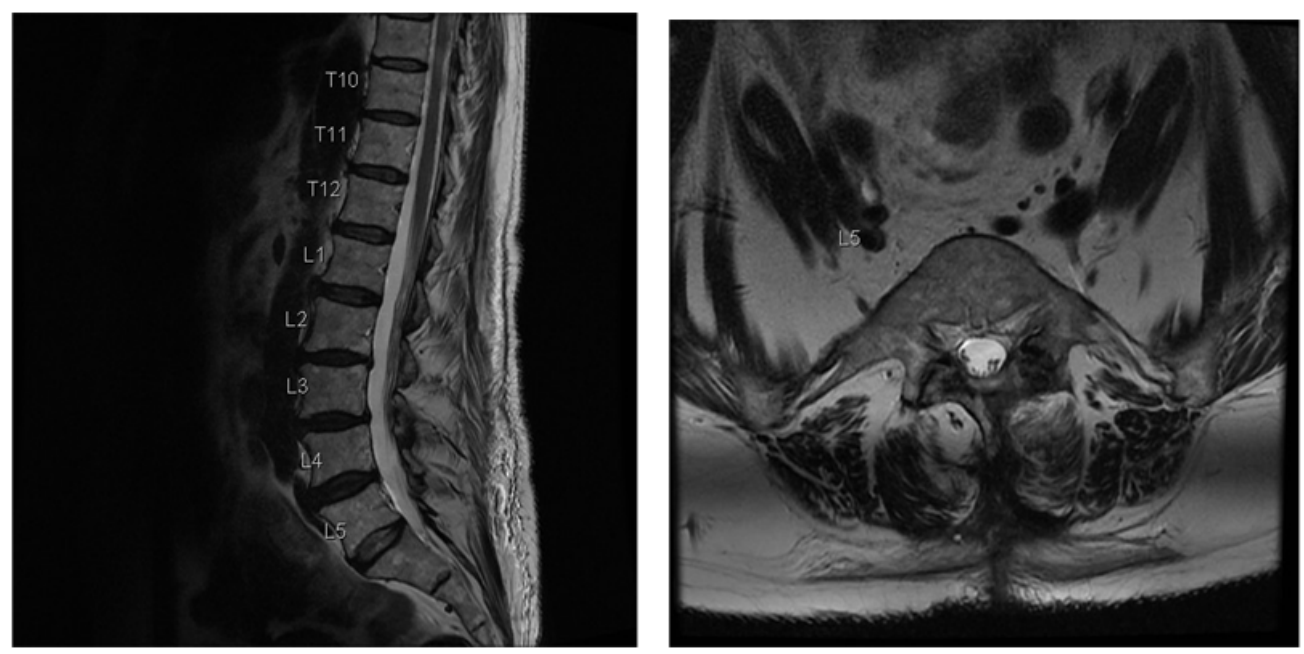

Figure 3. T2-weighted MR images of the lumbar spine in sagittal and transaxial planes 3 weeks after surgery. The hematoma has completely resolved. Patients consent obtained. 
The effect of metamizol on coagulation has been controversial in the last decades. In contrast to classical COX inhibitors, metamizol is a stronger inhibitor of platelet aggregation [5], but has no anti-inflammatory effect and low gastrointestinal toxicity, indicating a different mode of action [2]. The classical NSAIDs act by competing with arachidonic acid for binding to the cyclooxygenase active site. Metamizol and its active metabolites target the initiation of catalytic reaction of both COX isoforms [2] and cause a reversible inhibition of COX activity.

Bozzo et al. [5] compared the prohaemhorragic effect of different NSAIDs (dipyrone/metamizol, ibuprofen, ketorolac and aspirin) under experimental conditions. They performed perfusion studies and demonstrated that aspirin showed the most remarkable effects on platelet function under experimental conditions, closely followed by metamizol [5].

Clinical studies also showed an in vivo effect on platelet function. Graff et al. compared the effect of parecoxib and metamizol on platelet aggregation in patients undergoing meniscectomy in a double blind randomized trial [7] and found a significant lower platelet aggregation and TxB2 formation in the metamizol group. A related study [8] showed that the administration of COX inhibiting agents in general and of metamizol in particular led to hypoaggregability in the first days of subarachnoid hemorrhage (SAH). Furthermore, Lunas at al. evaluated the risk of gastrointestinal bleeding associated with analgetic drugs and NSAIDs and showed a small increased risk of upper gastrointestinal bleeding for metamizol (odds ratio 2,6), which was similar in magnitude to that observed with low dose aspirin [9].

At the beginning our patient recovered quickly and analgesia-especially opioids-were reduced, replaced by raised metamizol intake. Our patient received $3000 \mathrm{mg}$ of metamizol in 3 single $1000 \mathrm{mg}$ doses on day 2 and 3 after operation.

The COX inhibitory effect of metamizol correlates strongly with the plasma concentration of the active metabolites of metamizol. It has been shown by measuring the coagulation induced thromboxane B2 formation that after administration of $1000 \mathrm{mg}$ of metamizol 97\% of COX 1 activity was blocked for several hours. Previous data suggest that an excess of 95\% inhibition of serum thromboxane B2 significantly affects platelet function. [10]. Compared with acetylsalicylic acid the platelet inhibition is nearly equally strong but reversible [3] [4] [10].

Recently a prospective randomized clinical trial that was designed to show the analgesic effect of magnesium in post-tonsillectomy patients showed no increased bleeding risk in patients receiving metamizol. Apart from the fact that it was not designed to show safety of metamizol, the administered dose was 500 mg two times per day. In this low dose no bleeding risk was found [11].

The acetylsalicylic acid medication was stopped 7 days before the operation was scheduled, following our routine protocol. Acetylsalicylic acid irreversibly inhibits platelet function for the duration of the life of the platelets which is known to be 10 days [12], so the 7 days might have been too short. The begin of symptoms on day 3 which is day 10 after acetylsalicylic acid has been stopped suggests no influence on this bleeding. Interestingly recent publications suggest an antagonist effect of metamizol and acetylic salicylic acid. It has been demonstrated that metamizol has a high potential to attenuate or prevent antiplatelet effect of aspirin [13]. Docking studies suggest that NSAIDs forming hydrogen bonds with Ser530, Arg120, Tyr385 and other amino acids of the COX-1 hydrophobic channel interfere with antiplatelet activity of aspirin while non interfering NSAIDs do not form relevant hydrogen bond interactions within the aspirin binding site [14]. The plasma half-life of aspirin is only $20 \mathrm{~min}$ in circulating blood. It is rapidly deacetylated and converted to salicylate in vivo. Salicylate does doesn't affect COX-1 or COX-2 activity. The long term effect of acetylsalicylic acid that is caused by irreversibly blocking COX 1 formation in platelets is lost. This time correlation has been demonstrated for acetylsalicylic acid [15]. This interaction has no impact on this setting.

Antiplatelet effect of metamizol and acetylsalicylic acid did not differ significantly in vitro. The observations may indicate a competitive interaction between the two drugs. In vivo experiments showed that intravenously administered metamizol was an effective antiplatelet drug and can be considered as a therapeutic alternative, when acetylsalicylic acid cannot be used in oral form [12] [16].

Radicular pain and preoperative neurological deficit of this patient recovered quickly, after stopping prohaemhorragic medication and administration of high doses of steroids to reduce the local edema. Reoperation was therefore not needed. The most likely reason for bleeding was neutralized by simply stopping the drug related prohaemhorragic effects. The time correlation of risen dose of metamizol and the symptom onset suggested a causal relationship. Admittedly none of the other mentioned reasons for the bleeding can be ruled out. The question if metamizol can contribute to a postsurgical bleeding remains unanswered. Fact is that metamizol is 
one of the least well documented analgesic drugs [1].

\section{Conclusions}

For ages metamizol was assumed to be "centrally acting” and apart from the rare agranulocytosis, it was free of side effects. Recent publications show strong efficacy of metamizol compared with paracetamol [17]. As it is assumed to be safe, the overall use of metamizol has multiplied in the last years [1].

The dose of metamizol in postoperative pain management is up to 4 times $1000 \mathrm{mg}$ a day, which is a quantity that clearly shows a platelet inhibiting effect [3]. Nevertheless it is used widely even in context with neurosurgery.

Further prospective studies are needed to document the dose related benefit or disadvantage of this old drug especially in context with postoperative pain management after neurosurgical interventions.

\section{References}

[1] Theiler, R. and Dudler, J. (2013) Drug Therapy of Pain: Reason Overwhelmed by Emotion? Revue méDicale Suisse, 9, 1846-1853.

[2] Pierre, S.C., Schmidt, R., Brenneis, C., Michaelis, M., Geisslinger, G. and Scholich, K. (2007) Inhibition of Cyclooxygenases by Dipyrone. British Journal of Pharmacology, 151, 494-503. http://dx.doi.org/10.1038/sj.bjp.0707239

[3] Hinz, B., Cheremina, O., Bachmakov, J., Renner, B., Zolk, O., Fromm, M.F. and Brune, K. (2007) Dipyrone Elicits Substantial Inhibition of Peripheral Cyclooxygenases in Humans: New Insights into the Pharmacology of an Old Analgesic. The FASEB Journal, 21, 2343-2351. http://dx.doi.org/10.1096/fj.06-8061com

[4] Lampl, C. and Likar, R. (2014) Metamizole (Dipyrone): Mode of Action, Drug-Drug Interactions, and Risk of Agranulocytosis. Der Schmerz, 28, 584-590. http://dx.doi.org/10.1007/s00482-014-1490-7

[5] Bozzo, J., Escolar, G., Hernández, M.R., Galán, A.M. and Ordinas, A. (2001) Prohemorrhagic Potential of Dipyrone, Ibuprofen, Ketorolac, and Aspirin: Mechanisms Associated with Blood Flow and Erythrocyte Deformability. Journal of Cardiovascular Pharmacology, 38, 183-190.

[6] Arbeitsgemeinsschaft der wissenschaftlichen, medizinischen Fachgesellschaften (2009) S3-Leitlinie: Prophylaxe der venösen Thromboembolie (VTE).

[7] Graff, J., Arabmotlagh, M., Cheung, R., Geisslinger, G. and Harder, S. (2007) Effects of Parecoxib and Dipyrone on Platelet Aggregation in Patients Undergoing meniscectomy: A Double-Blind, Randomized, Parallel-Group Study. Clinical Therapeutics, 29, 438-447. http://dx.doi.org/10.1016/S0149-2918(07)80082-8

[8] Parkhutik, V., Lago, A., Tembl, J.I., Rubio, C., Fuset, M.P., Vallés, J., Santos, M.T. and Moscardo, A. (2012) Influence of COX-Inhibiting Analgesics on the Platelet Function of Patients with Subarachnoid Hemorrhage. Journal of Stroke \& Cerebrovascular Disease, 21, 755-759. http://dx.doi.org/10.1016/j.jstrokecerebrovasdis.2011.04.002

[9] Lanas, A., Serrano, P., Bajador, E., Fuentes, J. and Sáinz, R. (2003) Risk of Upper Gastrointestinal Bleeding Associated with Non-Aspirin Cardiovascular Drugs, Analgesics and Nonsteroidal Anti-Inflammatory Drugs. European Journal of Gastroenterology \& Hepatology, 15, 173-178. http://dx.doi.org/10.1097/00042737-200302000-00011

[10] Reilly, I.A. and FitzGerald, G.A. (1987) Inhibition of Thromboxane Formation in Vivo and ex Vivo: Implications for Therapy with Platelet Inhibitory Drugs. Blood, 69, 180-186.

[11] Tugrul, S., Degirmenci, N., Eren, S.B., Dogan, R., Veyseller, B. and Ozturan, O. (2014) Analgesic Effect of Magnesium in Post-Tonsillectomy Patients: A Prospective Randomised Clinical Trial. European Archives of Oto-Rhino-Laryngology, August 2014. http://dx.doi.org/10.1007/s00405-014-3219-8

[12] Altman, R., Luciardi, H.L., Muntaner, J. and Herrera, R.N. (2004) The Antithrombotic Profile of Aspirin. Aspirin Resistance, or Simply Failure? Thrombosis Journal, 2, 1. http://dx.doi.org/10.1186/1477-9560-2-1

[13] Hohlfeld, T., Zimmermann, N., Weber, A.-A., Jessen, G., Weber, H., Schrör, K., Höltje, H.-D. and Ebel, R. (2008) Pyrazolinone Analgesics Prevent the Antiplatelet Effect of Aspirin and Preserve Human Platelet Thromboxane Synthesis. Journal of Thrombosis and Haemostasis, 6, 166-173. http://dx.doi.org/10.1111/j.1538-7836.2007.02800.x

[14] Saxena, A., Balaramnavar, V.M., Hohlfeld, T. and Saxena, A.K. (2013) Drug/Drug Interaction of Common NSAIDs with Antiplatelet Effect of Aspirin in Human Platelets. European Journal of Pharmacology, 721, 215-224.

http://dx.doi.org/10.1016/j.ejphar.2013.09.032

[15] Anzellotti, P., Capone, M.L., Jeyam, A., Tacconelli, S., Bruno, A., Tontodonati, P., Di Francesco, L., Grossi, L., Renda, G., Merciaro, G., Di Gregorio, P., Price, T.S., Garcia Rodriguez, L.A. and Patrignani, P. (2011) Low-Dose Naproxen Interferes with the Antiplatelet Effects of Aspirin in Healthy Subjects: Recommendations to Minimize the Functional Consequences. Arthritis \& Rheumatism, 63, 850-859. http://dx.doi.org/10.1002/art.30175 
[16] Papp, J., Sandor, B., Vamos, Z., Botor, D., Toth, A., Rabai, M., Kenyeres, P., Cseplo, P., Juricskay, I., Mezosi, E., Koller, A. and Toth, K. (2014) Antiplatelet Effect of Acetylsalicylic Acid, Metamizole and Their Combination-in Vitro and in Vivo Comparisons. Clinical Hemorheology Microcirculation, 56, 1-12.

[17] Oreskovic, Z., Bicanic, G., Hrabac, P., Tripkovic, B. and Delimar, D. (2014) Treatment of Postoperative Pain after Total Hip Arthroplasty: Comparison between Metamizol and Paracetamol as Adjunctive to Opioid Analgesics-Prospective, Double-Blind, Randomised Study. Archives of Orthopaedic and Trauma Surgery, 134, 631-636.

http://dx.doi.org/10.1007/s00402-014-1979-7 